\title{
AVALIAÇÃO DE METODOLOGIA DE FÁCIL ANÁLISE PARA A DETERMINAÇÃO DE DADOS DE EQUILÍBRIO EM DUAS FASES AQUOSAS DO SISTEMA PEG (1500 $\left.\mathrm{g} \mathrm{mol}^{-1}\right)+$ ÁGUA + SAL
}

\author{
J. C. NASCIMENTOํㅛ, G. R. dos SANTOS 2 \\ ${ }^{1}$ Universidade Federal do Pará, Faculdade de Engenharia Química \\ ${ }^{2}$ Universidade Federal do Pará, Faculdade de Engenharia de Alimentos \\ E-mail para contato: julianenasciment@gmail.com
}

\begin{abstract}
RESUMO - Os sistemas aquosos bifásicos são uma variante da extração líquidolíquido que favorece a estabilidade de moléculas devido a que suas fases são formadas predominantemente por água. Sua obtenção pode ser dada pela combinação de um polímero e um sal. Assim, o objetivo deste trabalho é determinar dados experimentais do equilíbrio líquido-líquido em sistemas contendo PEG $1500+\mathrm{K}_{3} \mathrm{PO}_{4}+\mathrm{H}_{2} \mathrm{O}$ à temperatura de $30^{\circ} \mathrm{C}$. Para tal, foram determinados o limite de solubilidade do sistema, pelo método de ponto de turvação; e as linhas de amarração, pelo método indireto, consistente na quantificação dos componentes em cada fase mediante o uso das curvas de calibração e binodal. Estes dados foram comparados com a literatura, a fim de verificar o efeito do tipo de sal no equilíbrio de fases.
\end{abstract}

\section{INTRODUÇÃO}

Sistemas aquosos bifásicos (SABs) constituem uma excelente alternativa para separação, concentração e purificação de moléculas bioativas, pois favorecem a estabilidade de moléculas com origem em sistemas biológicos (COSTA, 2010; BARBOSA, 2013), devido as fases destes serem formados predominantemente $(60-95 \%$ em massa) por água, além de apresentarem vantagens por se tratar de um processo de baixo custo, alta seletividade e com possibilidade de reciclagem dos reagentes (CESAR, 2000; SILVA \& LOH, 2006). Os SABs têm como princípio a utilização de duas fases aquosas imiscíveis, que podem ser obtidas pela combinação de dois polímeros hidrossolúveis ou de um polímero e um sal; estudos mais recentes têm utilizado líquido iônico (LI) e sal inorgânico (BARBOSA, 2013).

Recentemente, têm sido relatados dados de equilíbrio para sistemas de duas fases contendo Polietileno Glicol (PEG) e sal inorgânico, como fosfatos e sulfatos (ZHANG et al., 2013; AZEVEDO, 2015). Entretanto, ainda são poucos os dados de equilíbrio liquido-liquido disponíveis na literatura para os sistemas contendo PEG e Fosfato de Potássio (MORALES et al., 2010; HAGHTALAB \& MOKHTARANI, 2004). Assim, este trabalho visa determinar dados experimentais do equilíbrio líquido-líquido em sistemas contendo Polietileno glicol (1500 $\left.\mathrm{g} \mathrm{mol}^{-1}\right)$, água e Fosfato de potássio à temperatura de $30^{\circ} \mathrm{C}$, por meio do método indireto. 


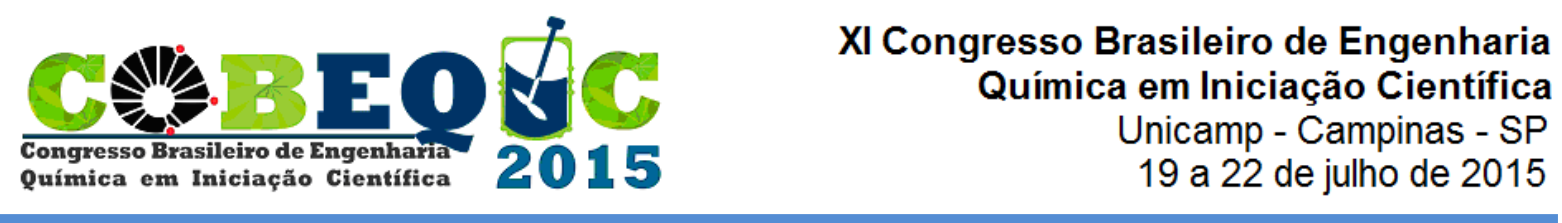

\section{MATERIAIS E MÉTODOS}

\subsection{Materiais}

Para a formação do equilíbrio liquido-liquido foram utilizados PEG de massa molar $1500 \mathrm{~g} \mathrm{~mol}^{-1}$ (Fluka Analytical), Fosfato de Potássio (Sigma-Aldrich, 98\%) e água destilada. Estes foram manuseados mediante o uso de balança analítica (Quimis, Q500L210C, precisão de 0,0001 g), banho termostático (B. Braun Biotech International, Thermomix BM) e refratômetro digital (Table Digital Refractometer, TDR095C).

\subsection{Métodos}

Curva Binodal: Inicialmente, foram preparadas soluções aquosas de PEG a 50\% (p/p) e Sal a $25 \%(\mathrm{p} / \mathrm{p})$. Estas foram utilizadas para a determinação experimental da curva binodal, pelo método de ponto de turvação (Cloud Point), que consiste em adicionar sal a uma solução de PEG ou PEG a uma solução de sal, até a obtenção de uma solução bifásica, seguindo da adição gota a gota de água destilada, em agitação constante, até a detecção de uma região levemente turva. Este procedimento foi realizado em um banho termostático, a temperatura de $30^{\circ} \mathrm{C}$. Vale ressaltar a importância de manter a temperatura constante, uma vez que a mudança desta provoca o deslocamento da curva binodal, mudando assim a região bifásica, o que implica em uma maior ou menor concentração do sal e do polímero necessários para a formação do sistema.

Linhas de Amarração: A determinação das linhas de amarração ocorreu pela produção de soluções com uma concentração situada dentro da região de miscibilidade parcial, onde os componentes requeridos foram pesados e homogeneizados cuidadosamente. A mistura foi mantida em repouso, em banho termostático, por $17 \mathrm{hs}$ à temperatura de $30^{\circ} \mathrm{C}$. Após este tempo, amostras de ambas as fases foram coletadas e suas composições foram determinadas pelo método indireto, que se baseia em informações das curvas de calibração e binodal.

Curvas de Calibração: A construção da curva de calibração se deu pelo preparo de soluções de concentrações conhecidas sobre a curva binodal e realização da leitura do Brix. Estes dados de concentração e Brix foram correlacionados usando uma equação de segundo grau, de modo a se obter um $\mathrm{R}^{2}$ próximo a unidade.

\section{RESULTADOS E DISCUSSÃO}

\subsection{Curva Binodal}

A Figura 1 representa a curva binodal, a qual demarca a região em que o sistema deixa de ser monofásico e passa a ser bifásico. Composições localizadas na região abaixo da curva formam sistemas com uma fase, enquanto que na região acima, formam sistemas com duas fases homogêneas. Vale ressaltar que, os componentes presentes em maior quantidade nas fases inferior e superior são representados no eixo das abscissas e das ordenadas, respectivamente.

Na Figura 1 é apresentada também a curva binodal do sistema PEG 1500 + LiSO4 + $\mathrm{H} 2 \mathrm{O}$ à $30^{\circ} \mathrm{C}$ e pôde ser observado uma pequena redução da região bifásica. Isto ocorre devido 


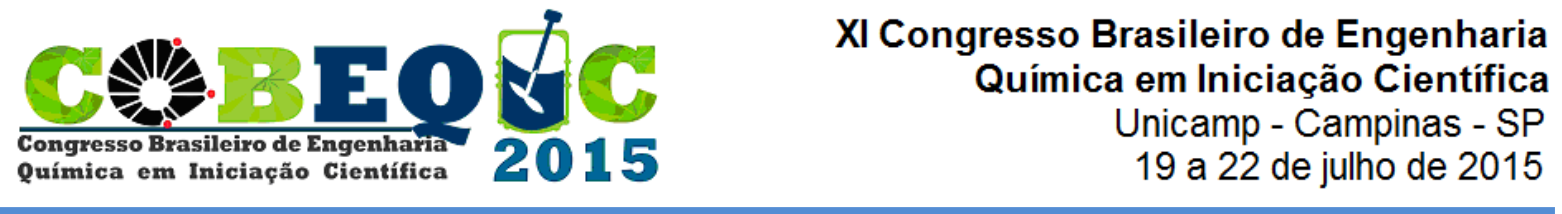

existirem pequenas diferenças nos coeficientes de partição de diferentes sais, ou seja, diferentes íons possuem diferentes afinidades pelas fases (SILVA, 2007).

Figura 1- Curva binodal do Sistema PEG 1500+ K3PO4+H2O à $30^{\circ} \mathrm{C}$.

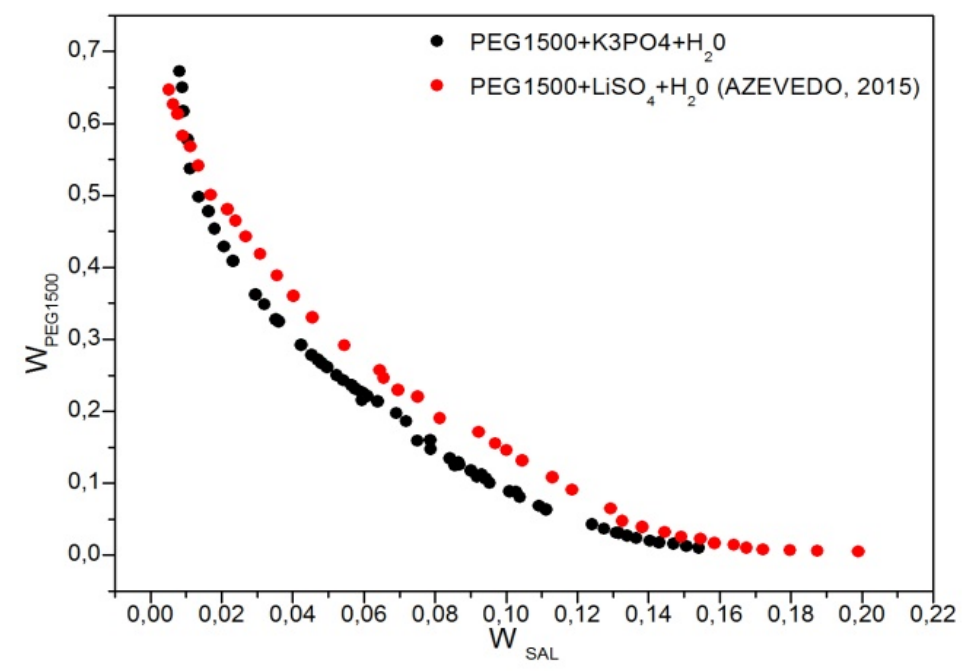

A partir dos dados mostrados na Figura 1, foi possível estimar uma equação exponencial, que descrevesse a curva binodal, dada por:

$W_{1}=e^{-0,34851-19,91821 \times W_{2}-6,8286 \times W_{2}^{2}}$

cujo coeficiente de correlação $\mathrm{R}^{2}$ foi igual a 0,98492 . Esta foi utilizada na determinação das composições das fases juntamente com as equações obtidas pelo balanço de massa, dada por:

$W_{1}+W_{2}+W_{3}=1$

e pela curva de calibração. $\mathrm{W}_{1}$ corresponde a fração mássica de $\mathrm{PEG}, \mathrm{W}_{2}$ a fração mássica de sal e $\mathrm{W}_{3}$ a fração mássica de água.

\subsection{Curva de calibração}

Após a reprodução de alguns pontos da curva binodal, realizou-se uma análise em triplicata do Brix, que é uma propriedade física que varia conforme a concentração dos componentes da solução. As frações mássicas de cada componente bem como as médias do Brix e seus desvios estão apresentados na Tabela 1.

Os dados apresentados na Tabela 1 foram representados na Figura 2, a qual relaciona a variação dos valores de Brix com as diferentes composições de PEG1500 e $\mathrm{K}_{3} \mathrm{PO}_{4}$. Estes dados foram correlacionados a uma equação, dada abaixo, e apresentou um coeficiente de correlação $\mathrm{R}^{2}$ de 0,99779 .

$$
B R I X=-24,20214+193,69057 \times W_{1}+317,83459 \times W_{2}-113,54396 \times W_{1}^{2}-183,49033 \times W_{2}^{2}
$$




\begin{tabular}{ccccc|ccccc}
\hline & $\mathrm{W}_{\mathrm{PEG}}$ & $\mathrm{W}_{\mathrm{K} 3 \mathrm{PO} 4}$ & $\mathrm{~W}_{\mathrm{H} 2 \mathrm{O}}$ & $\mathrm{BRIX}$ & & $\mathrm{W}_{\mathrm{PEG}}$ & $\mathrm{W}_{\mathrm{K} 3 \mathrm{PO} 4}$ & $\mathrm{~W}_{\mathrm{H} 2 \mathrm{O}}$ & BRIX \\
\hline 1 & 0,6485 & 0,0089 & 0,3427 & $52,23 \pm 0,21$ & 6 & 0,1600 & 0,0784 & 0,7616 & $24,50 \pm 0,10$ \\
2 & 0,5778 & 0,0103 & 0,4118 & $49,40 \pm 0,10$ & 7 & 0,0847 & 0,1106 & 0,8047 & $19,70 \pm 0,10$ \\
3 & 0,4319 & 0,0206 & 0,5475 & $40,73 \pm 0,21$ & 8 & 0,0377 & 0,1323 & 0,8300 & $18,13 \pm 0,06$ \\
4 & 0,3195 & 0,0350 & 0,6455 & $32,87 \pm 0,15$ & 9 & 0,0171 & 0,1499 & 0,8330 & $18,50 \pm 0,10$ \\
5 & 0,2094 & 0,0612 & 0,7294 & $25,67 \pm 0,31$ & - & --- & --- & --- & --- \\
\hline
\end{tabular}

Figura 2- Curva de Calibração do Sistema PEG1500 + K3PO4 + H2O à $30^{\circ} \mathrm{C}$.

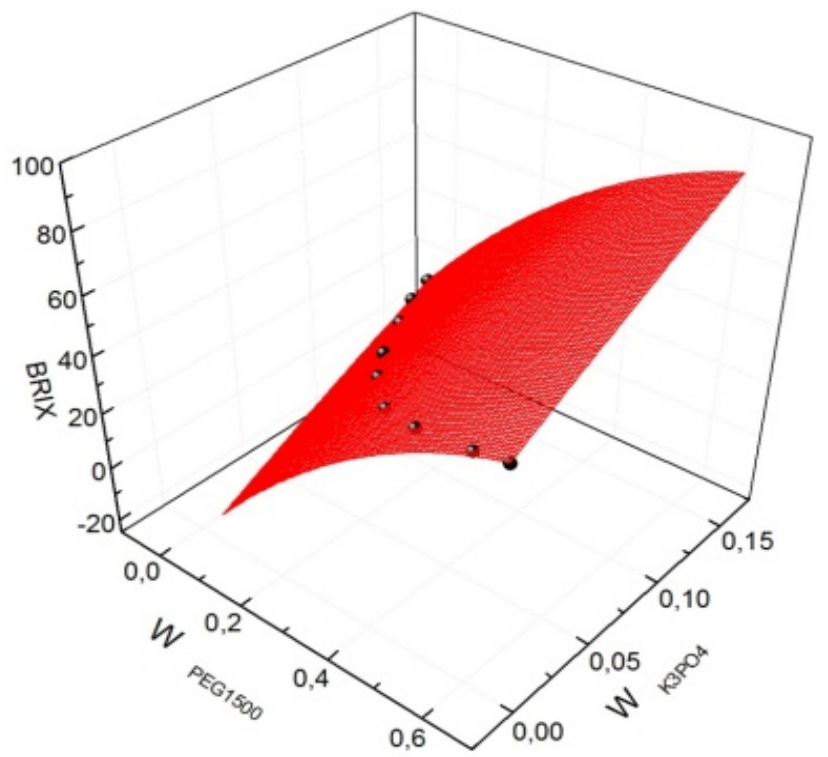

\subsection{Linhas de amarração}

Os resultados das análises de Brix e seus respectivos desvios de cada uma das fases coletadas são apresentados na Tabela 2.

Tabela 2- Brix das fases superior e inferior

\begin{tabular}{ccc}
\hline & \multicolumn{2}{c}{ BRIX } \\
\hline Células & Fase Superior & Fase Inferior \\
1 & $31,57 \pm 0,12$ & $19,17 \pm 0,06$ \\
2 & $35,27 \pm 0,12$ & $21,13 \pm 0,45$ \\
3 & $41,50 \pm 0,20$ & $23,80 \pm 0,10$ \\
4 & $44,23 \pm 0,23$ & $28,83 \pm 0,15$ \\
\hline
\end{tabular}


Os dados de equilíbrio obtidos são apresentados na Tabela 3. As frações mássicas de cada componente presente em cada fase foram determinadas pela resolução do sistema citado anteriormente, por meio da utilização de programas computacionais.

Tabela 3- Dados experimentais do equilíbrio líquido-líquido do sistema PEG1500 $+\mathrm{K}_{3} \mathrm{PO}_{4}+$ $\mathrm{H}_{2} \mathrm{O}$ a $30^{\circ} \mathrm{C}$

\begin{tabular}{c|ccc|ccc|cccc}
\hline \multicolumn{3}{c}{ Global } & \multicolumn{4}{c}{ Fase Superior } & \multicolumn{3}{c}{ Fase Inferior } \\
\hline Células & $\mathrm{W}_{1}$ & $\mathrm{~W}_{2}$ & $\mathrm{~W}_{3}$ & $\mathrm{~W}_{1}$ & $\mathrm{~W}_{2}$ & $\mathrm{~W}_{3}$ & $\mathrm{~W}_{1}$ & $\mathrm{~W}_{2}$ & $\mathrm{~W}_{3}$ \\
1 & 0,2339 & 0,0621 & 0,7040 & 0,2838 & 0,0450 & 0,6711 & 0,0442 & 0,1330 & 0,8228 \\
2 & 0,2723 & 0,0683 & 0,6594 & 0,3327 & 0,0373 & 0,6300 & 0,0306 & 0,1499 & 0,8195 \\
3 & 0,3119 & 0,0741 & 0,6140 & 0,4248 & 0,0253 & 0,5500 & 0,0209 & 0,1672 & 0,8119 \\
4 & 0,3490 & 0,0797 & 0,5713 & 0,4721 & 0,0200 & 0,5078 & 0,0161 & 0,1907 & 0,7943 \\
\hline
\end{tabular}

A Figura 3 mostra a composição global da mistura, bem como a composição das duas fases em equilíbrio. Qualquer conjunto de pontos que pertençam à região bifásica e que estejam sobre a mesma linha de amarração fornecerão fases superiores que possuirão propriedades termodinâmicas intensivas iguais (densidade, volume molar, entalpia molar, etc.), entretanto, sendo distintas as suas variáveis termodinâmicas extensivas (massa, volume, etc). Aplica-se o mesmo raciocínio para as fases inferiores formadas a partir de composições globais localizadas sobre uma mesma linha de amarração (SILVA \& LOH, 2006).

Figura 3- Diagrama de Equilíbrio de Fases do Sistema PEG1500 + K3PO4 + H2O a $30^{\circ} \mathrm{C}$.

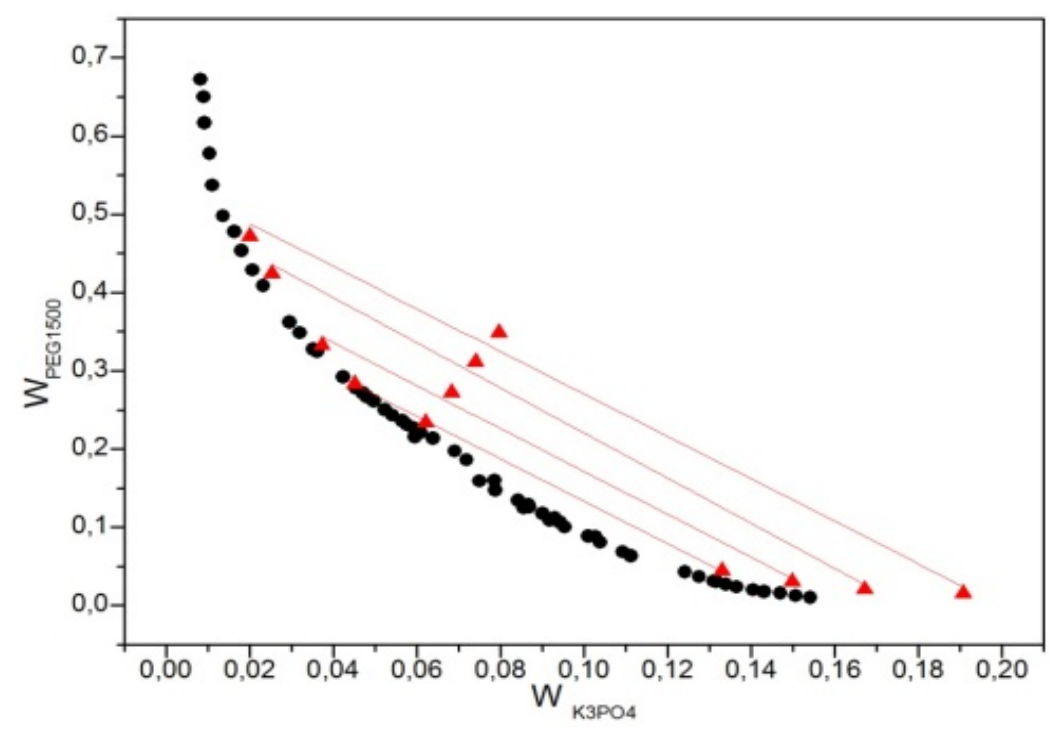

\section{CONCLUSÃO}

A curva de solubilidade do sistema PEG1500 $+\mathrm{K}_{3} \mathrm{PO}_{4}+\mathrm{H}_{2} \mathrm{O}$ a temperatura de $30^{\circ} \mathrm{C}$ foi determinada pelo método de cloud-point, delimitando a região monofásica e bifásica. Quando comparada com dados da literatura verificou-se uma pequena redução da região bifásica, 


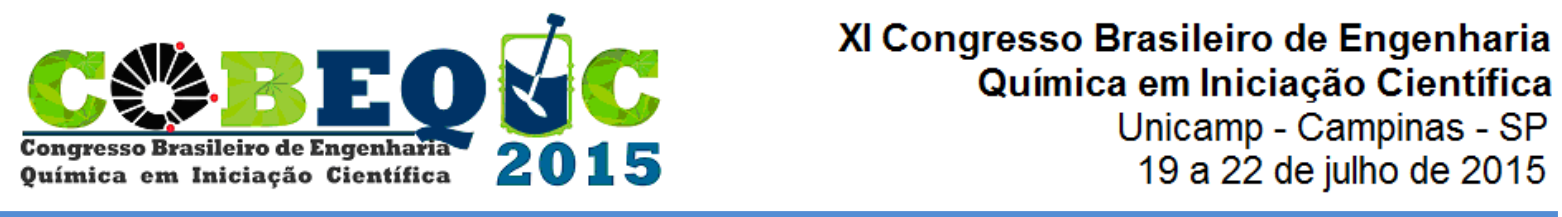

indicando assim a influência do sal no sistema, visto que diferentes íons possuem diferentes afinidades pelas fases. Utilizando equações geradas pelos dados deste trabalho, a saber: Curva binodal, Curva de Calibração e Balanço de massa; foi possível relacionar as composições dos componentes nas fases superior e inferior, determinando assim as linhas de amarração.

\section{REFERÊNCIAS BIBLIOGRÁFICAS}

AZEVEDO, C. M. Determinação experimental e modelagem termodinâmica do sistema em duas fases aquosas polietileno glicol 1500, sulfato de lítio a 303,15k. 2015. 39 f. TCC (Graduação) - Curso de Engenharia Química, Universidade Federal do Pará, Belém, 2015.

BARBOSA, A. A. Obtenção de dados de equilíbrio de sistemas aquosos formados por peg, sulfato e água e partição de antocianina do jamelão. 2013. 74 f. Dissertação (Mestrado) - Curso de Engenharia de Alimentos, Universidade Estadual do Sudoeste da Bahia, Itapetinga, 2013.

CESAR, A. C. W. Otimização dos parâmetros de Extração Líquido- Líquido em Duas Fases Aquosas na Recuperação da Bromelina Presente no Abacaxi. 2000. 67 f. Dissertação (Mestrado) - Curso de Faculdade de Engenharia Química, Universidade Estadual de Campinas, Campinas, 2000.

COSTA, A. R. da. Participação de lactoferrina em sistemas aquosos bifásicos. 2010. 56 f. Dissertação (Mestrado) - Curso de Engenharia de Alimentos, Universidade Estadual do Sudoeste da Bahia, Itapetinga, 2010.

HAGHTALAB, A.; MOKHTARANI, B. The new experimental data and a new thermodynamic model based on group contribution for correlation liquid-liquid equilibria in aqueous two-phase systems of PEG and (K2HPO4 or Na2SO4). Fluid Phase Equilibria, v. 215, p.151-161, fev. 2004.

MORAlES, J. W.; GAlleGUILlOS, H. R.; GRABER, T. A.; HERNANDEZ-LUIZ F. Activity coefficients of $\mathrm{LiCl}$ in (PEG $4000+$ water) at $\mathrm{T}=(288.15,298.15$, and 308.15) K. The Journal Of Chemical Thermodynamics. v. 42, p. 1255-1260. maio 2010.

SILVA, L. H. M. da; LOH, W. Sistemas aquosos bifásicos: fundamentos e aplicações para partição/purificação de proteínas. Quimica Nova, v. 29, n. 6, p.1345-1351, 14 jun. 2006.

SILVA, C. A. S. Partição do Glicomacropeptídeo usando sistemas aquosos bifásicos. 2007, 57f. Dissertação (Mestrado)- Curso de Universidade Federal de Viçosa, Viçosa, 2007.

ZHANG, W.; HU, Y.; WANG, Y.; HAN, J.; NI, L.; WU, Y. et al. Liquid-liquid equilibrium of aqueous two-phase systems containing poly(ethylene glycol) of different molecular weights and several ammonium salts at 298.15 K. Thermochimica Acta, v. 560, p. $47-$ 54. mar. 2013. 